\title{
ARTICLE
}

\section{Differences in DNA methylation profiles by histologic subtype of paediatric germ cell tumours: a report from the Children's Oncology Group}

\author{
Lindsay A. Williams ${ }^{1}$, Lauren Mills ${ }^{2}$, Anthony J. Hooten ${ }^{1}$, Erica Langer ${ }^{1}$, Michelle Roesler ${ }^{1}$, A. Lindsay Frazier ${ }^{3}$, Mark Krailo ${ }^{4}$, \\ Heather H. Nelson ${ }^{5,6}$, Jessica Bestrashniy ${ }^{1}$, James F. Amatruda ${ }^{7}$ and Jenny N. Poynter ${ }^{1,6}$
}

\begin{abstract}
BACKGROUND: Abnormal DNA methylation may be important in germ cell tumour (GCT) aetiology, as germ cells undergo complete epigenetic reprogramming during development. GCTs show differences in global and promoter methylation patterns by histologic subtype. We conducted an epigenome-wide study to identify methylation differences by GCT histology.

METHODS: Using the Illumina HumanMethylation450K array we measured methylation in 154 paediatric GCTs (21 germinomas/ seminomas/dysgerminoma, 70 yolk sac tumours [YST], 9 teratomas, and 54 mixed histology tumours). We identified differentially methylated regions (DMRs) between GCT histologies by comparing methylation beta values.

RESULTS: We identified 8,481 DMRs (FWER < 0.05). Unsupervised hierarchical clustering of individual probes within DMRs resulted in four high level clusters closely corresponding to tumour histology. Clusters corresponding to age, location, sex and FFPE status were not observed within these DMRs. Germinomas displayed lower levels of methylation across the DMRs relative to the other histologic subtypes. Pathway analysis on the top $10 \%$ of genes with differential methylation in germinomas/seminomas/ dysgerminoma compared to YST suggested angiogenesis and immune cell-related pathways displayed decreased methylation in germinomas/seminomas/dysgerminoma relative to YST.
\end{abstract}

CONCLUSIONS: Paediatric GCT histologies have differential methylation patterns. The genes that are differentially methylated may provide insights into GCT aetiology including the timing of GCT initiation.

British Journal of Cancer (2018) 119:864-872; https://doi.org/10.1038/s41416-018-0277-5

\section{INTRODUCTION}

Germ cell tumours (GCTs), arising from totipotent primordial germ cells (PGC), are histologically heterogeneous tumours found in males and females in both gonadal and extragonadal locations. The main histologic subtypes of GCTs in the paediatric and adolescent population include: germinoma arising in the brain, yolk sac tumour (YST), seminoma/dysgerminoma, and teratomas (mature and immature). ${ }^{1}$ Tumours of mixed histology, that includes embryonal carcinoma and/or choriocarcinoma are also common. ${ }^{1}$ The predominant histologic subtype and tumour location differ by sex and age. ${ }^{2}$ GCTs are more frequently diagnosed in males when considering children aged 0-19 years.,4 Among males $<5$ years of age, tumours are equally distributed among extragonadal locations and the testes. ${ }^{3}$ In males aged $<5$ years, the most common histology is YST and/or teratomas; conversely, GCTs in adolescent males are most often of mixed histologic subtypes and/or seminoma and are more frequently found in the testes. ${ }^{2,3,5-7}$ In contrast, for females, tumours are predominantly extragonadal prior to the age of 5 and then switch to a gonadal predominance throughout adolescence. As with males, the most common histology among females $<5$ years of age is generally YST and/or teratomas, whereas in adolescence, the most common histologic subtypes are dysgerminoma and teratoma. $^{2}$ Extragonadal tumours are more common in the paediatric age range compared to adolescent or young adult tumours, and are thought to arise from abnormal migration of PGCs during embryogenesis. ${ }^{8,9}$ As germ cells undergo extensive epigenetic reprogramming during the embryonic and early developmental periods, ${ }^{6,10-12}$ understanding differences in methylation patterns between histologic subtypes may shed light on aetiologic variation by histology as we work toward identifying important windows of exposure.

While strong risk factors for GCTs have yet to be identified, methylation changes in PGCs may be a biologic mechanism of tumour initiation and growth ${ }^{11}$ and may be influenced by environmental exposures during critical windows of development. Germ cells undergo two cycles of demethylation and reestablishment of methylation as part of normal development during

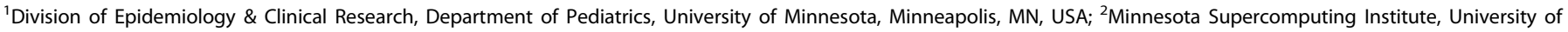

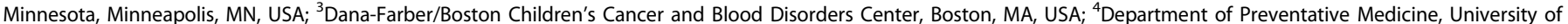

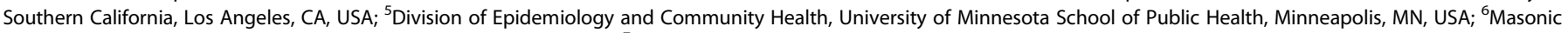

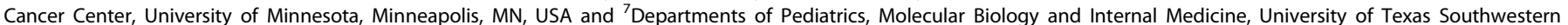
Medical Center, Dallas, TX, USA

Correspondence: Jenny N. Poynter (poynt006@umn.edu)

Received: 26 April 2018 Revised: 6 September 2018 Accepted: 10 September 2018

Published online: 5 October 2018 
embryogenesis and development, which may present relevant windows of susceptibility whereby environmental exposures to toxicants or stress may have lasting impacts on DNA methylation. ${ }^{12}$ Anomalous DNA methylation contributes to carcinogenesis by regulating gene expression and may be especially relevant for GCT development due to the extensive epigenetic reprogramming that occurs during embryogenesis and development. ${ }^{12,13}$ In general, paediatric tumours are less likely than adult cancers to result from the accumulation of mutations or DNA damage possibly due to the short latency period from birth to cancer diagnosis. $^{6,14}$ Therefore, other mechanisms such as aberrant DNA methylation in GCT development may play an essential role in GCT initiation and progression, particularly among paediatric GCTs.

The histologic subtypes of GCTs display a gradient of global methylation with seminomas nearly lacking global methylation and less differentiated tumours (e.g., embryonal carcinoma) experiencing lower levels of global methylation relative to more differentiated tumours (e.g., teratoma). ${ }^{15-23}$ Differences in promoter methylation and expression have also been reported by GCT histology, particularly within key germ cell developmental signalling pathways including the BMP/TGF beta pathways. ${ }^{7,19}$ Differences in methylation patterns at imprinted loci between histologic subtypes of paediatric GCTs have also been reported. ${ }^{20}$ To further characterise differences in methylation between the histologic subtypes of paediatric GCTs, we conducted an epigenome-wide study to examine differences in methylation by histologic subtype among a histologically diverse set of 154 paediatric tumours including germinomas/seminomas/dysgerminomas, teratomas, yolk sac and mixed histologic subtype from gonadal, extragonadal and intracranial locations.

\section{METHODS}

Samples

Tumour specimens were available for 154 paediatric GCTs (21 germinomas/seminoma/dysgerminoma, 54 mixed, 9 teratomas, 70 yolk sac tumours [YST]) collected from three sources (University of Texas Southwestern Medical Centre, Dallas, TX USA; Children's Oncology Group; Boston Children's Hospital, Boston, MA USA). Patient and tumour characteristic data were abstracted from medical records and pathology reports including patient age (0-10, 11-19 years), patient sex (male, female, gonadal dysgenesis, unknown), tumour histology (germinomas/seminoma/dysgerminoma, teratoma, yolk sac [pure yolk sac or teratoma and yolk sac histology], mixed/other [choriocarcinoma and embryonal carcinoma]) and tumour location (intracranial, extragonadal, gonadal [testis, ovary]). Informed written consent was obtained prior to sample collection at the treating institution. All study protocols were approved by the University of Minnesota Institutional Review Board.

\section{Methylation analysis}

Prior to methylation analysis, $500 \mathrm{ng}$ genomic DNA was treated with sodium bisulphite using the EZ DNA Methylation Kit (Zymo Research, Orange, CA) according to the manufacturer's protocol. Genome-wide methylation analysis was performed using the Infinium HumanMethylation450 BeadChip array (Illumina, San Diego, (A) at the University of Minnesota Genomics Centre following Illumina's standard protocol. For FFPE samples, we used the Infinium FFPE DNA Restore Kit (Illumina, Inc.) to repair DNA prior to array methylation analysis. All DNA samples were assessed for quality prior to analysis and duplicates were included for 19 samples to control for chip variation. The methylation status of a specific CpG site was calculated as the variable $\beta$, which is the ratio of the fluorescent signal from a locus specific probe to the methylated allele to the sum of the fluorescent signals of locus specific probes for both methylated and unmethylated alleles. ${ }^{24}$ These values range from 0 (unmethylated) to 1 (fully methylated).

Quality control and determination of methylation levels Array quality control and analysis was performed in R (v3.4.1) using the minfi package $(\mathrm{v} 1.18 .2){ }^{25,26}$ Arrays were checked for incomplete bisulphite conversion and overall beta value distributions. Outlier arrays were removed from the analysis. There was no evidence of batch effects similar to what has been reported elsewhere. ${ }^{27}$ Probes with known SNPs were removed from the study and the methylation status of the $X$ and $Y$ chromosome in each sample was checked against the reported sex to look for mislabelling or other array errors. FFPE $(n=50,32.5 \%)$ and frozen ( $n=104,67.5 \%)$ tumour samples were not distinctly different from one another (Fig. 1a) as has been observed between tumour samples in a recent validation study. ${ }^{28}$ Beta values were calculated for each probe using quantile normalisation.

Principal component analysis (PCA)

PCA was performed using quantile normalised methylation (beta) values for or all samples with known histology and FFPE status via the prcomp function in $\mathrm{R}$ (v3.4.1) using the covariance matrix (scale $=$ FALSE).
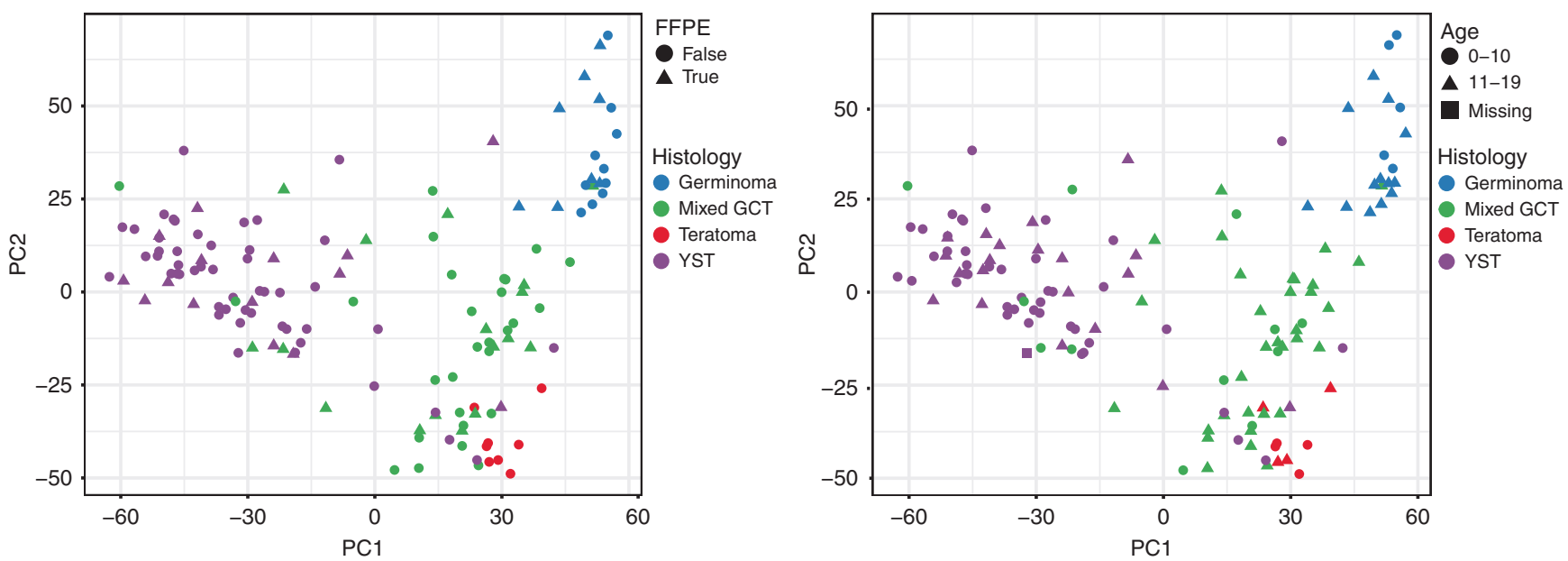

Fig. 1 Principal component analysis was performed using quantile normalised methylation values from all probes for all samples with histology and FFPE status that had arrays after removing duplicate and control samples. a Point colours indicate tumour histology while shape indicates FFPE status. b Point colours indicate tumour histology while shape indicates patient age. Germinoma includes germinoma, seminoma and dysgerminoma 
DMR analysis

Differentially methylated regions (DMRs) were identified using bumphunter from the minfi package and quantile normalised beta values. DMRs were identified using generalised linear models and a smoothing technique as part of the bumphunter algorithm. The use of DMRs as the informative measure of differential methylation allowed us to capture groups of $\mathrm{CpGs}$ within the same region that possess the same methylation patterns while also capturing individual CpG loci that are differentially methylated. Only arrays with information for FFPE and histology were used during DMR tests. Family-wise error rates (FWER) for the DMR tests were computed using the bootstrap null method $(B=200)$. The DMR cutoff was 0.2 or $20 \%$, as used in other methylation analyses and validation studies. ${ }^{2,29-31}$ The closest gene was identified for each DMR using bedtools closestBed and hg19 RefSeq gene annotations.

\section{Clustering}

The heatmap was created in $\mathrm{R}$ (v3.4.1) using the pheatmap package on the quantile normalised methylation (beta) values. Hierarchal clustering using complete Euclidean distance was used to cluster samples (columns) and probe/DMR methylation values (rows) into similar groups. Samples with FFPE status and histology were included in the DMR calculations (18 germinomas/seminomas/dysgerminoma, 45 mixed tumours, 8 teratoma and 65 YST).

\section{Ingenuity pathway analysis}

The top $10 \%$ of genes based on differences in methylation values that showed increased methylation in germinomas/seminomas/ dysgerminoma relative to YST $(n=300)$ and the top $10 \%(n=550)$ showing increased methylation in YST over germinomas/seminomas/dysgerminoma were entered into IPA to identify pathways of interest. The $10 \%$ of genes with differential methylation in germinomas/seminomas/dysgerminoma vs. YST were selected as a way to reduce the number of genes included in the pathway analysis, which is generally recommended to be conducted using a few hundred genes. ${ }^{32}$ The difference in methylation beta value between the two histologic subtypes was entered into IPA and pathways were identified using the IPA Knowledge Base gene list. Pathways with only one gene listed were omitted.
Ratio of DMRs to total probes

The total number of probes with an absolute value for the differences in methylation beta value $>0.2$ between germinomas/ seminomas/dysgerminoma compared to YST was calculated. If

$\beta_{\text {germinomas/seminomas/dysgerminoma }}-\beta_{Y S T}$ was less than -0.2 these probes were considered to have increased methylation in YST relative to germinomas/seminomas/dysgerminoma. If $\beta_{\text {germinomas/ }}$ seminomas/dysgerminoma $-\beta_{\text {YST }}$ was $>0.2$ these probes were considered to have increased methylation in germinomas/seminomas/ dysgerminoma compared to YST. The ratio of DMRs to total probes was calculated by taking the number of probes with the appropriate difference in beta value divided by the total number of probes per chromosome.

Identification of DMRs with extreme differences in methylation by histology

We also identified DMRs with extreme differences in methylation in germinomas/seminomas/dysgerminoma compared with YST as these may be genes of aetiologic importance. For this analysis, we classified $\beta<0.3$ as hypomethylated and $\beta>0.7$ as hypermethylated. We then selected DMRs that were hypomethylated in germinomas/seminomas/dysgerminoma and hypermethylated in YST and DMRs with $\beta>0.7$ (hypermethylated) in germinomas/ seminomas/dysgerminoma and $\beta<0.3$ (hypomethylated) in YST. The differential $\beta$ was calculated as $\beta$ germinomas/seminomas/dysgermi-

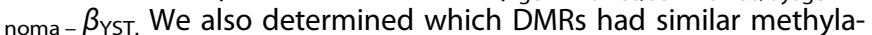
tion status in both histologies defined as hypomethylated $(n=1)$ or hypermethylated $(n=22)$ in both germinomas/seminomas/ dysgerminoma and YST.

\section{Statistical analysis}

Fisher's exact tests were used to identify significant differences in patient age (0-10, 11-19 years), sex (male, female, gonadal dysgenesis), and tumour location (intracranial, extragonadal, ovary, testes) by histology (germinomas/seminomas/dysgerminoma, mixed, teratoma, YST). Two-sided $p$-values for tests of statistical significance with an alpha of 0.05 were estimated. Patient and tumour characteristic analyses were done in SAS version 9.4 (Cary, North Carolina).

\begin{tabular}{|c|c|c|c|c|c|}
\hline \multicolumn{6}{|l|}{ Age } \\
\hline $0-<11$ & $5(23.8)$ & $13(24.1)$ & $5(55.6)$ & $49(71.0)$ & $72(47.1)$ \\
\hline $11-19$ & $16(76.2)$ & $41(75.9)$ & $4(44.4)$ & $20(29.0)$ & $81(52.9)$ \\
\hline \multicolumn{6}{|l|}{ Sex } \\
\hline Male & $7(33.3)$ & $37(68.5)$ & $3(37.5)$ & $29(47.5)$ & $76(52.8)$ \\
\hline Female & $12(57.1)$ & $17(31.5)$ & $5(62.5)$ & $32(52.5)$ & $66(45.8)$ \\
\hline Gonadal dysgenesis & $2(9.5)$ & $0(0.0)$ & $0(0.0)$ & $0(0.0)$ & $2(1.4)$ \\
\hline Missing & 0 & 0 & 1 & 9 & 10 \\
\hline Testis & $4(21.1)$ & $33(61.1)$ & $0(0.0)$ & $24(38.1)$ & $61(42.1)$ \\
\hline Missing & 2 & 0 & 0 & 7 & 9 \\
\hline
\end{tabular}

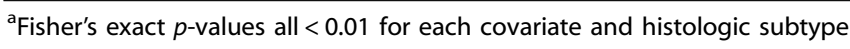




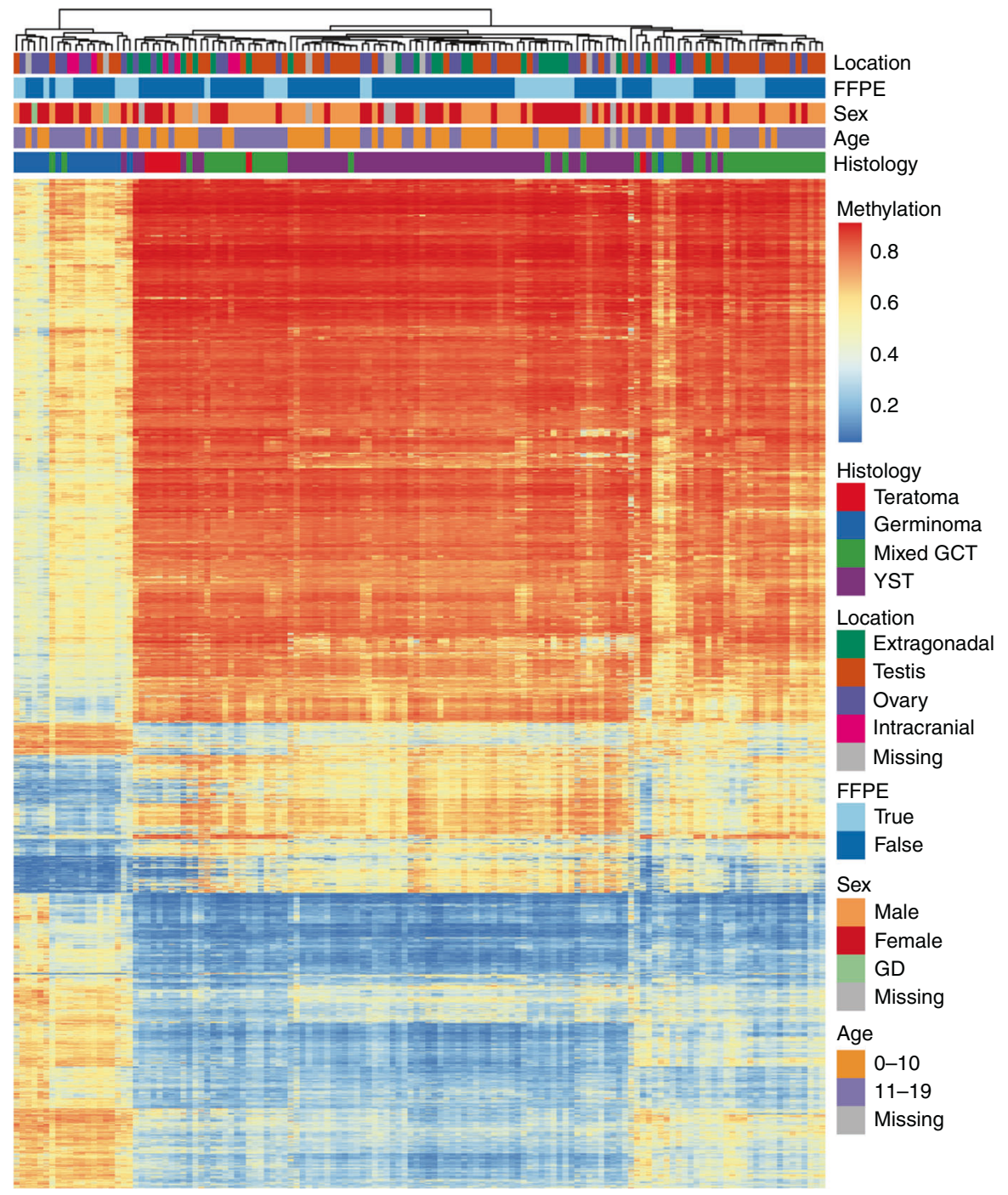

Fig. 2 Unsupervised, hierarchical clustering was performed on the average methylation value for all the probes within each DMR for each sample. Colour coding of tumour characteristics is at the top and normalised methylation values are shown in each row from low methylation (blue) to higher methylation (red). Germinoma includes germinoma, seminoma and dysgerminoma

\section{RESULTS}

Patient and tumour characteristics

There were significant differences in age, sex, and tumour location by GCT histologic subtype (Table 1). When considering age category, $76 \%$ of both germinomas/seminoma/dysgerminoma and mixed tumours were diagnosed among children aged 11-19 years, teratomas were equally distributed by age, and $71 \%$ of yolk sac tumours (YST) were among children aged $0-10$ years $(p<0.01)$. Approximately $60 \%$ of germinomas/seminoma/ dysgerminoma and teratomas, $32 \%$ of mixed tumours and $53 \%$ of YST were diagnosed among females $(p<0.01)$. Tumour location varied significantly by GCT histologic subtype $(p<0.01)$ with $58 \%$ of germinomas/seminoma/dysgerminoma diagnosed in the ovary (dysgerminoma), $61 \%$ of mixed tumours diagnosed in the testes, teratomas were more evenly distributed among tumour locations, and no YSTs were intracranial but were more frequently gonadal in location.

Histologic subtype clustering independent of age

PCA of the gene methylation beta values for all probes was used to visualise methylation differences by tumour histology, sex (results not shown) and age group. When we evaluated tumour histology, principal component (PC) 1 discriminated between YST and all other histologic subtypes while PC2 separated germinomas/seminomas/dysgerminoma from the mixed tumours and teratoma, which overlapped one another (Fig. 1a). PC1 explained $25.5 \%$ of the variance between samples while $12.7 \%$ of the variance was explained by PC2. There was no noticeable clustering pattern by age (Fig. 1b).

Differential methylation by histologic subtype

Comparison of methylation levels across the genome between GCT histologic subtypes identified 8481 differentially methylated regions (DMRs; FWER $<0.05)$. When stratifying by age group $(0-10$ vs. 11-19 years), 2228 DMRs (FWER $<0.05)$ were identified; however, these differences did not persist when tumour histology and age were included as variables in the model used to test for differential methylation indicating that the age-specific DMRs were not preserved between different histologic subtypes.

Unsupervised, hierarchal clustering was conducted using the methylation beta values for each probe within the differentially methylated regions (DMRs) identified between the histological subtypes (Fig. 2). Among the pure histologic subtypes 17/18 germinomas/seminoma/dysgerminoma and 52/65 YST consistently clustered together. In general, teratoma and mixed GCTs clustered with their respective subtype, but showed some 
868

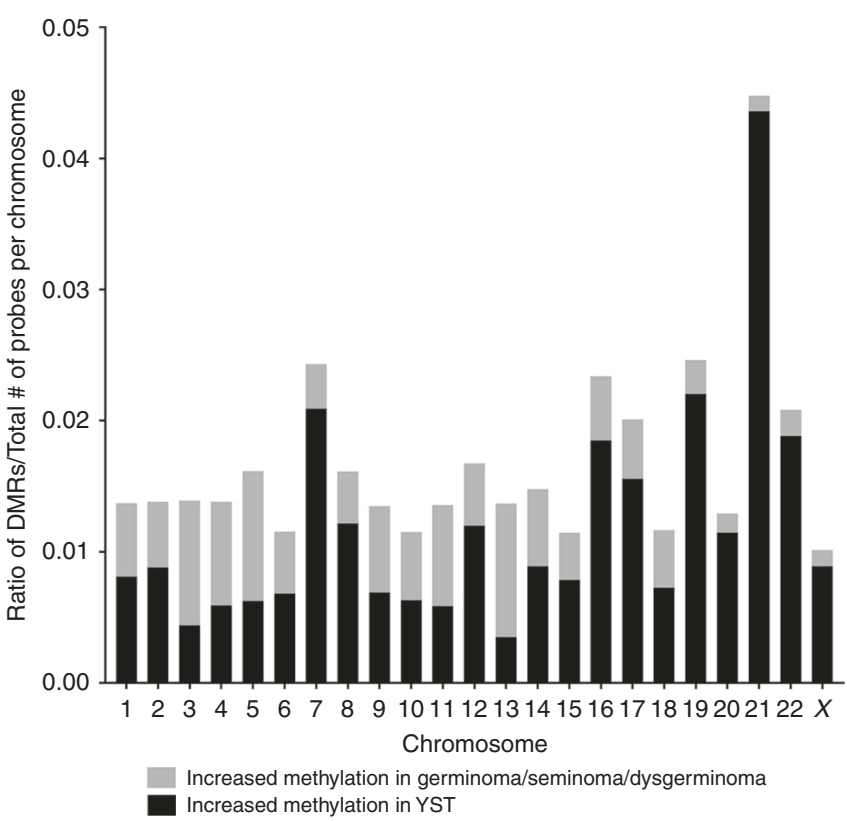

Fig. 3 Ratio of DRMs to the total number of probes for DMRs with increased methylation in germinoma relative to YST (grey) and increased in YST relative to germinoma/seminoma/dysgerminoma (black)

variability in clustering location. Germinomas/seminoma/dysgerminoma displayed a unique methylation pattern characterised by lower levels of epigenome-wide methylation relative to other tumour types, which were more similar to one another. Unlike histologic subtype, age, sex, tumour location, and FFPE status were not associated with specific clusters.

Differential methylation between germinomas/seminomas/ dysgerminoma and YST

Comparing the pure histologic subtypes of germinomas/seminoma/dysgerminoma and YST, we identified pathways differentially associated with histologic subtype. Of the 8481 DMRs identified between all histologic subtypes, 2969 loci showed increased methylation in germinomas/seminoma/dysgerminoma relative to YST and 5512 loci showed decreased methylation in germinomas/seminoma/dysgerminoma relative to YST.

We compared the ratio of the number of DMRs with a difference in methylation beta value greater than the absolute value of 0.2 to the total number of probes on each chromosome to determine whether DMRs were evenly distributed across the genome (Fig. 3). The highest ratio of DMRs to total probes were identified on chromosomes 7, 16, 17, 19, 21 and 22, with a particularly notable ratio for increased methylation in YST relative to germinomas/seminomas/dysgerminoma on chromosome 21. Germinomas/seminoma/dysgerminoma had a higher ratio of DMRs to total probes representing increased methylation relative to YST on chromosomes 3,5 and 13. We observed one gene (ADAP1) that was hypermethylated in both germinomas/seminomas/dysgerminoma and YST (both $\beta>0.7$ ) and 22 genes (results not shown) that were hypomethylated in both germinomas/seminomas/dysgerminoma and YST (both $\beta<0.3$ ); four of these still reached our threshold as DMRs (differential $\beta>0.2$ ), but did not meet the threshold for inclusion in the pathway analysis. The remaining DMRs had average differential beta values of 0.29 (standard deviation $=0.07$ ). Additionally, we identified 51 genes that were hypomethylated $(\beta<0.3)$ in one histologic subtype and hypermethylated $(\beta>0.7)$ in the other histologic subtype (Supplemental Table 2).
Pathway analysis of DMRs between germinomas/seminomas/ dysgerminoma and YST

Ingenuity pathway analysis (IPA) was used to identify pathways that were enriched in the top $10 \%$ of loci based on the absolute difference in beta values with differential methylation in germinomas/seminoma/dysgerminoma and YST. Compared to YST, among germinomas/seminomas/dysgerminoma there were 346 pathways with decreased methylation and 246 pathways with increased methylation. Pathways with $p$-values $<0.01$ are presented in Table 2 (additional pathways in Supplemental Table 1). The top pathways with decreased methylation in germinomas/ seminomas/dysgerminoma relative to YST include the TWEAK signalling, tec kinase signalling, and RAR activation pathways. The highly significant pathways with increased methylation in germinomas/seminoma/dysgerminoma relative to YST include the axonal guidance signalling, signalling by rho family GTPases, and the breast cancer regulation by stathmin 1 pathways.

\section{DISCUSSION}

In our histologically diverse epigenome-wide study of 154 paediatric GCTs, we identified 8481 regions with differential methylation by histologic subtype, which is in-line with the number of DMRs identified in a study of paediatric neuroblastoma samples. ${ }^{33}$ When overall methylation levels were summarised via PCA, germinomas/seminoma/dysgerminoma and YST were clustered tightly into two distinct groups and were the most distant from each other along the axis representing principal component 1. Age, sex, location and FFPE status did not create distinct groups when visualising the first two principal components. In unsupervised, hierarchical clustering, germinomas/seminoma/dysgerminoma formed a strong cluster while the remaining tumour types showed more variability in clustering by histology. Importantly, these clustering patterns did not appear to be driven by FFPE status, sex, age and tumour location. Germinomas/seminoma/ dysgerminoma displayed a strong tendency toward epigenomewide hypomethylation relative to the other histologic subtypes. Finally, when comparing methylation profiles of germinomas/ seminoma/dysgerminoma and YST, we observed increased methylation among YSTs particularly in established tumour suppressor genes and immune regulatory, cell proliferation and angiogenesis pathways.

While there is considerable variation in age at diagnosis by GCT histologic subtype, in our analysis of paediatric and adolescent GCTs, histology rather than age was the major source of variation across the samples as visualised by the first two principal components. This is consistent with previous analyses of methylation in intracranial $\mathrm{GCTs}^{23}$ and gene expression in paediatric $\mathrm{GCTs}^{34}$ where molecular differences were predicted more accurately by histology than patient age. We observed consistent clustering of germinomas/seminoma/dysgerminoma cases based on methylation beta values and this subtype displayed lower levels of methylation relative to other subtypes, which was similarly reported among intracranial GCTs. ${ }^{23}$ Conversely, we and others have reported that YST and teratomas generally clustered together and displayed high global methylation relative to the other histologic subtypes. ${ }^{22}$ While there are few studies on differential methylation by histologic subtype in paediatric GCTs, studies of adult testicular GCT have reported similar differences in methylation patterns between seminoma and nonseminoma tumour subtypes. ${ }^{22,35}$

There are established differences in response to treatment by histologic subtype, with nonseminomas, such as YST, exhibiting poorer responses to radio- and chemotherapy. ${ }^{36}$ Therefore, characterising differentially methylated genes and pathways between YST and germinomas/seminomas/dysgerminoma may aid in understanding therapy resistance and identify potentially druggable targets. Studies of paediatric GCTs have reported that 
Differences in DNA methylation profiles by histologic subtype of...

Table 2. Canonical pathways represented among the top $10 \%$ of decreased and increased methylated genes from differentially methylated regions in germinoma relative to yolk sac tumours ( $p$-values $<0.01$ )

\begin{tabular}{|c|c|c|}
\hline \multicolumn{3}{|l|}{ Decreased methylation in germinoma ${ }^{a}$ compared to YST } \\
\hline TWEAK signalling & TRAF3, TRAF2, TNFSF12, CASP8, CASP7, TRAF1 & 0.0001 \\
\hline Tec kinase signalling & $\begin{array}{l}\text { PTK2, PIK3R3, TNFSF12, GNAO1, RHOT2, GNB2, PIK3CD, JAK3, GNB1L, TNF, FNBP1, } \\
\text { PRKCZ }\end{array}$ & 0.0001 \\
\hline Sphingosine-1-phosphate signalling & PTK2, PIK3R3, PLCD3, PDGFA, ADCY3, RHOT2, PIK3CD, CASP8, FNBP1, CASP7 & 0.0002 \\
\hline Role of NFAT in cardiac hypertrophy & $\begin{array}{l}\text { PIK3R3, PLCD3, NKX2-5, HDAC7, ADCY3, GNB2, PRKACA, IGF1R, HAND1, PIK3CD, } \\
\text { GNB1L, PRKCZ }\end{array}$ & 0.0004 \\
\hline Molecular mechanisms of cancer & $\begin{array}{l}\text { RAPGEF1, RHOT2, ADCY3, PRKCZ, PIK3R3, PTK2, NF1, CDK20, GNAO1, E2F1, PRKACA, } \\
\text { PIK3CD, CASP8, JAK3, NOTCH1, FNBP1, CASP7, LRP1 }\end{array}$ & 0.001 \\
\hline NF- $\mathrm{KB}$ signalling & PIK3R3, TRAF3, TRAF2, PRKACA, IGF1R, PIK3CD, LTBR, CASP8, TNFRSF1B, TNF, PRKCZ & 0.001 \\
\hline $\begin{array}{l}\text { Role of macrophages, fibroblasts and endothelial cells in } \\
\text { rheumatoid arthritis }\end{array}$ & $\begin{array}{l}\text { TRAF3, PDGFA, PRKCZ, PIK3R3, PLCD3, TRAF2, APC2, GNAO1, PIK3CD, LTBR, SOST, } \\
\text { TNFRSF1B, TNF, LRP1, TRAF1 }\end{array}$ & 0.001 \\
\hline Type I diabetes mellitus signalling & CD247, CD28, TRAF2, HLA-A, GAD1, CASP8, TNFRSF1B, TNF & 0.002 \\
\hline CXCR4 signalling & PTK2, PIK3R3, GNAO1, ADCY3, RHOT2, GNB2, PIK3CD, GNB1L, FNBP1, PRKCZ & 0.002 \\
\hline Lymphotoxin $\beta$-receptor signalling & PIK3R3, TRAF3, TRAF2, PIK3CD, LTBR, TRAF1 & 0.002 \\
\hline Death receptor signalling & TRAF2, TNFSF12, LMNA, CASP8, TNFRSF1B, TNF, CASP7 & 0.002 \\
\hline Thrombin signalling & PTK2, PIK3R3, PLCD3, GNAO1, ADCY3, RHOT2, GNB2, PIK3CD, GNB1L, FNBP1, PRKCZ & 0.002 \\
\hline Myc-mediated apoptosis signalling & PIK3R3, YWHAG, IGF1R, PIK3CD, CASP8, PRKCZ & 0.002 \\
\hline TNFR2 signalling & TRAF2, TNFRSF1B, TNF, TRAF1 & 0.003 \\
\hline Colorectal cancer metastasis signalling & PIK3R3, GRK2, ADCY3, RHOT2, GNB2, PRKACA, PIK3CD, JAK3, GNB1L, TNF, FNBP1, LRP1 & 0.004 \\
\hline CREB signalling in neurons & PIK3R3, PLCD3, GRM3, GNAO1, ADCY3, GNB2, PRKACA, PIK3CD, GNB1L, PRKCZ & 0.004 \\
\hline Relaxin signalling & PIK3R3, PDE9A, GNAO1, ADCY3, GNB2, PRKACA, PIK3CD, GNB1L, PRKCZ & 0.004 \\
\hline Goq signalling & PIK3R3, GRK2, CSK, RHOT2, GNB2, PIK3CD, GNB1L, FNBP1, PRKCZ & 0.004 \\
\hline \multicolumn{3}{|l|}{ Increased methylation in germinoma relative to YST } \\
\hline Axonal guidance signalling & $\begin{array}{l}\text { NTNG1, EFNB2, SRGAP3, BMP4, GLIS1, ARHGEF7, GNA12, C9orf3, ADAM19, PFN2, HHIP, } \\
\text { KLB, GNG7, PRKCB }\end{array}$ & 0.0005 \\
\hline Signalling by Rho family GTPases & MAP3K9, CDH1, GNA12, ARHGEF7, PIP4K2B, ARHGEF18, ARHGEF3, KLB, GNG7 & 0.002 \\
\hline Breast cancer regulation by stathmin 1 & CAMK1D, ARHGEF7, ARHGEF18, ITPR1, ARHGEF3, KLB, GNG7, PRKCB & 0.002 \\
\hline RhoGDI signalling & CDH1, GNA12, ARHGEF7, PIP4K2B, ARHGEF18, ARHGEF3, GNG7 & 0.003 \\
\hline TR/RXR activation & F10, PDE3B, PFKP, THRB, KLB & 0.004 \\
\hline Coagulation system & F10, F3, SERPINF2 & 0.006 \\
\hline Thrombin signalling & CAMK1D, GNA12, ITPR1, ARHGEF3, KLB, GNG7, PRKCB & 0.007 \\
\hline Guanosine nucleotides degradation III & NT5C2, ACPP & 0.007 \\
\hline Relaxin signalling & PDE3B, GNA12, PDE4D, NPR2, KLB, GNG7 & 0.007 \\
\hline Urate biosynthesis/inosine 5'-phosphate degradation & NT5C2, ACPP & 0.009 \\
\hline
\end{tabular}

Wnt pathway regulator, ${ }^{37}$ the adenomatous polyposis coli (APC) gene, was methylated in YST but not germinoma ${ }^{19,38,39}$ leading to activation of Wnt signalling. ${ }^{40,41}$ In our study, we observed hypermethylation of Wnt pathway regulator, APC2, in YST relative to germinomas/seminoma/dysgerminoma (results not shown). We observed increased methylation for ASCL2, a Wnt target gene, ${ }^{42}$ and NPY, an activator of Wnt pathway proteins, ${ }^{43}$ in YST relative to germinomas/seminoma/dysgerminoma as others have reported (results not shown). ${ }^{19}$ Additionally, in YST we observed hypermethylation of previously identified genes suspected to be tumour suppressors including: HOXA9, SCGB3A1, IRF5, CASP8 and LTB4R. ${ }^{19,20}$ In agreement with Amatruda et al. ${ }^{20}$ who reported 
differential methylation between YST compared to the other histologic subtypes, we observed differential methylation for YST and germinomas/seminoma/dysgerminoma for RASSF1, SCGB3A1, HOXA9, FGF3, PDGFRB, NPY, ASCL2, CDK10 and GUCY2D. In a previous analysis, Noor et al. ${ }^{44}$ identified 72 genes with differential methylation and correlated gene expression in seminoma and YST from adult TGCT cell lines and paediatric GCT tumour samples. ${ }^{34}$ In our analysis, we observed differential methylation in 15/72 genes identified by Noor et al. This suggests that there may be a number of important genes that are dysregulated in both paediatric and adult YSTs relative to seminomas. Collectively, these findings may highlight potential target genes and pathways for drug development to improve outcomes for YST in particular.

When we used IPA to identify pathways over-represented from the identified DMRs in germinomas/seminomas/dysgerminoma compared to YST, we identified pathways with both decreased and increased methylation. Pathways with significantly decreased methylation in germinomas/seminoma/dysgerminoma relative to YST include the TWEAK, tec kinase signalling, RAR activation and a number of immune cell-related pathways. The TWEAK signalling pathway is hypothesised to contribute to tumour proliferation and angiogenesis ${ }^{45}$ while the tec kinase signalling pathway plays a role in the activation of T- and B-cells. ${ }^{46,47}$ The RAR activation pathway plays an important role in developmental differentiation of cells. ${ }^{48}$ Various immune cell-related pathways had decreased methylation in germinomas/seminoma/dysgerminoma vs. YST. While a smaller number of pathways had increased methylation in germinomas/ seminoma/dysgerminoma compared to YST, these pathways did not appear to be related to immune signalling or immune cell development as observed for the pathways with decreased methylation in germinomas/seminoma/dysgerminoma relative to YST. Identified pathways with increased methylation in germinomas/seminoma/dysgerminoma include the axonal guidance signalling, signalling by rho family GTPases, and the breast cancer regulation by stathmin 1 pathways. The role of these specific pathways in the development of one histologic subtype of GCT over another remains unclear, but they have been associated with tumour development by contributing to cell proliferation, cell migration and angiogenesis. ${ }^{49-51}$

We identified a number of chromosomes with a higher ratio of DMRs to total probes in YST compared to germinomas/seminoma/ dysgerminoma, particularly for chromosomes 7, 16, 17, 19, 21 and 22. In the literature, there are reports of copy number alterations and gains of chromosomes in GCTs, which may explain the increased methylation we observed in YST compared to germinomas/seminoma/dysgerminoma. There is a reported gain of $17 q$ in embryonic cell lines ${ }^{52}$ and testicular nonseminoma. ${ }^{53}$ Gains of chromosomes 7, 8, 17, 21, 22 in testicular germ cell tumours (TGCTs) and GCT case reports have also been described. ${ }^{53-56}$ Few studies have reported a gain in chromosome $19{ }^{56}$ where we observed many DMRs with increased methylation in YST vs. germinomas/seminoma/dysgerminoma. We observed the largest ratio of DMRs to total probes with increased methylation in YST relative to germinomas/seminoma/dysgerminoma for chromosome 21 . There are reports of an increased risk of GCTs, particularly testicular tumours, among males with Down's syndrome. ${ }^{57-59}$ When we identified genes that were hypomethylated $(\beta<0.3)$ in one histologic subtype and hypermethylated $(\beta>$ 0.7 in another histologic subtype, in contrast to our first analysis using an absolute value of 0.2 as a differential methylation cut-off, we found a smaller number of genes that were hypomethylated in one histologic subtype yet hypermethylated in the other. These findings highlight regions of the genome that may be important in GCT development.

Our findings should be viewed in light of the following limitations. While we present an epigenome-wide study of methylation from a large number of paediatric GCTs, we were unable to subset the mixed tumours and immature and mature teratomas based on their histologic components, as this information was not available for our cases. Similarly, we were restricted by sample size to examine germinoma, seminoma and dysgerminoma separately. We were unable to examine methylation profiles in association with treatment response or survival, as we did not have adequate outcome data for the participants. We did not have normal tissue samples available to allow comparison of normal and tumour tissue methylation. Finally, in the present study, we do not have gene expression data available to examine the correlation between methylation and expression for the genes of interest; therefore, while gene methylation often correlates with gene expression, ${ }^{60,61}$ exploring these relationships within histologic subtypes of paediatric GCTs remains to be completed. Interestingly, Amatruda and colleagues ${ }^{20}$ found negative correlations between gene methylation and expression for some genes among teratoma samples suggesting a mechanistic role for methylation in gene expression control in paediatric GCTs. Further, gene methylation may also be informative beyond the regulation of gene expression as it may also serve as a marker of exposure or outcome as discussed by Michels et al. ${ }^{62}$

In conclusion, we observed distinct differences in gene-specific methylation between the histologic subtypes of GCT, particularly between germinoma/seminoma/dysgerminoma and YST. While it has been hypothesised that GCTs arise from the PGC and the resulting tumour type depends on the maturity of the $P G C^{63}$ environmental exposures that impact tumour microenvironment may exert phenotype modifying pressures that encourage tumours in the gonads or extragonadal locations to develop into one histologic subtype over another under the control of DNA methylation. The data from our epigenome-wide study along with the findings of other research groups suggest that alteration of methylation patterns could be a primary mechanism of histologic subtype determination. We and others ${ }^{19,20}$ have observed hypermethylation of tumour suppressor genes in YST, suggesting a potential mechanism for tumour initiation and chemotherapeutic resistance. Future work investigating these methylation changes in histologic subtypes of GCTs compared to methylation in PGCs and other precursor cell types may be informative in understanding GCT aetiology and the timing of GCT initiation. Further, the identification of windows of susceptibility during the prenatal and early developmental period may shed light on exposures that impact DNA methylation and lead to risk reduction strategies.

\section{ACKNOWLEDGEMENTS}

This work was supported by the National Institutes of Health (grant numbers R01 CA151284 to J.N.P.; T32 CA099936 to L.A.W.; NCTN Operations Centre Grant [U10 CA180886]; and NCTN Statistics \& Data Centre Grant [U10CA180899]), and the Children's Cancer Research Fund, Minneapolis, MN.

\section{AUTHOR CONTRIBUTIONS}

L.A.W. interpreted the data, conducted literature review, drafted manuscript. L.M. analysed the data and assisted data interpretation. J.N.P. designed study, conceptualised analysis, interpreted data. A.J.H. and E.L. conducted the laboratory assays. M.R. is the study coordinator, supervised data collection. A.L.F,. J.F.A., M.K., H.H. $\mathrm{N}$, J.B. contributed to the conceptualisation of the study. All authors contributed to drafting, review and approval of the final manuscript.

\section{ADDITIONAL INFORMATION}

Supplementary information is available for this paper at https://doi.org/10.1038/ s41416-018-0277-5.

Competing interests: The authors declare no competing interests.

Availability of data and materials: Data may be made available upon a reasonable request to the corresponding author. 
Ethics approval and consent to participate: Informed written parental consent was obtained prior to sample collection at the treating institution. All study protocols were approved by the University of Minnesota Institutional Review Board.

Note: This work is published under the standard license to publish agreement. After 12 months the work will become freely available and the license terms will switch to a Creative Commons Attribution 4.0 International (CC BY 4.0)

\section{REFERENCES}

1. Olson, T. A., Schneider, D. T., Perlman, E. J. in Germ Cell Tumors: Principles and Practice in Pediatric Oncology. Sixth edn. (eds Pizzo, P. A., Poplack, D. G.) 1045-1067 (Lippincott Williams \& Wilkins, Philadelphia, PA, 2011)

2. Poynter, J. N., Amatruda, J. F. \& Ross, J. A. Trends in incidence and survival of pediatric and adolescent patients with germ cell tumors in the United States, 1975 to 2006. Cancer [Internet] 116, 612-625 (2010).

3. Poynter, J. N. in Pediatric Germ Cell Tumors: Epidemiology of Germ Cell Tumors, First edn (eds Frazier, A. L., Amatruda, J. F.) 17-36 (Springer-Verlag, Heidelberg New York Dordrecht London, 2014)

4. Hall, C., Ritz, B., Cockburn, M., Davidson, T. B. \& Heck, J. E. Risk of malignant childhood germ cell tumors in relation to demographic, gestational, and perinatal characteristics. Cancer Epidemiol. [Internet]. 46, 42-49 (2017).

5. Rakheja, D., Teot, L. A. in Pediatric Germ Cell Tumors: Pathology of Germ Cell Tumors. (eds Frazier, A. L., Amatruda, J. F.) 37-58 (Springer-Verlag, Heidelberg New York Dordrecht London, 2014).

6. Cusack, M. \& Scotting, P. DNA methylation in germ cell tumour aetiology: Current understanding and outstanding questions. Reproduction 146, R49-R60 (2013).

7. Fustino, N., Rakheja, D., Ateek, C. S., Neumann, J. C. \& Amatruda, J. F. BMP signaling activity distinguishes histologic subsets of pediatric germ cell tumors. Int. J. Androl. 34, e218-e233 (2011).

8. Marcotte, E. L. et al. Variants in BAK1, SPRY4, and GAB2 are associated with pediatric germ cell tumors: A report from the children's oncology group. Genes Chromosome Cancer 56, 548-558 (2017).

9. Schneider, D. T. et al. Multipoint imprinting analysis indicates a common precursor cell for gonadal and nongonadal pediatric germ cell tumors. Cancer Res. 61, 7268-7276 (2001).

10. Godmann, M., Lambrot, R. \& Kimmins, S. The dynamic epigenetic program in male germ cells: Its role in spermatogenesis, testis cancer, and its response to the environment. Microsc. Res. Tech. 72, 603-619 (2009).

11. Bussey, K. J et al. SNRPN methylation patterns in germ cell tumors as a reflection of primordial germ cell development. Genes Chromosome Cancer [Internet]. 32, 342-352 (2001)

12. Faulk, C. \& Dolinoy, D. C. Timing is everything: The when and how of environmentally induced changes in the epigenome of animals. Epigenetics 6 , 791-797 (2011)

13. Smallwood, S. A. \& Kelsey, G. De novo DNA methylation: A germ cell perspective. Trends Genet [Internet]. 28, 33-42 (2012).

14. Gröbner, S. N. et al. The landscape of genomic alterations across childhood cancers. Nature [Internet]. (2018) Available from: http://www.nature.com/ doifinder/10.1038/nature25480

15. Netto, G. J. et al. Global DNA hypomethylation in intratubular germ cell neoplasia and seminoma, but not in nonseminomatous male germ cell tumors. Mod. Pathol. 21, 1337-1344 (2008).

16. Lind, G. E. \& Skotheim, R. I. \& Lothe, R. A. The epigenome of testicular germ cell tumors. APMIS [Internet]. 115, 1147-1160 (2007).

17. Smiraglia, D. J. et al. Distinct epigenetic phenotypes in seminomatous and nonseminomatous testicular germ cell tumors. Oncogene 21, 3909-3916 (2002).

18. Looijenga, L. H. J., Gillis, A. J. M., Stoop, H., Biermann, K. \& Oosterhuis, J. W. Dissecting the molecular pathways of (testicular) germ cell tumour pathogenesis; from initiation to treatment-resistance. Int. J. Androl. 34, e234-e251 (2011).

19. Jeyapalan, J. N. et al. Methylator phenotype of malignant germ cell tumours in children identifies strong candidates for chemotherapy resistance. Br. J. Cancer [Internet]. 105, 575-585 (2011).

20. Amatruda, J. F. et al. DNA methylation analysis reveals distinct methylation signatures in pediatric germ cell tumors. BMC Cancer [Internet]. 13, 313 (2013).

21. Wermann, $\mathrm{H}$ et al. Global DNA methylation in fetal human germ cells and germ cell tumours: Association with differentiation and cisplatin resistance. J. Pathol. 221, 433-442 (2010)

22. De Jong, J., Weeda, S., Gillis, A. J. M., Oosterhuis, J. W. \& Looijenga, L. H. J. Differential methylation of the OCT3/4 upstream region in primary human testicular germ cell tumors. Oncol. Rep. [Internet]. 18, 127-132 (2007).

23. Fukushima, S. et al. Genome-wide methylation profiles in primary intracranial germ cell tumors indicate a primordial germ cell origin for germinomas. Acta Neuropathol. 133, 445-462 (2017)
24. Bibikova, M. et al. High-throughput DNA methylation profiling using universal bead arrays. Genome Res. 16, 383-393 (2006).

25. Aryee, M. J. et al. Minfi: A flexible and comprehensive Bioconductor package for the analysis of Infinium DNA methylation microarrays. Bioinformatics 30 1363-1369 (2014).

26. Aryee, M. J. et al. Accurate genome-scale percentage DNA methylation estimates from microarray data. Biostatistics 12, 197-210 (2011).

27. Forest, $M$. et al. Agreement in DNA methylation levels from the Illumina $450 \mathrm{~K}$ array across batches, tissues, and time. Epigenetics 13, 1-14 (2018).

28. Moran, B. et al. Assessment of concordance between fresh-frozen and formalinfixed paraffin embedded tumor DNA methylation using a targeted sequencing approach. Oncotarget [Internet]. 8, 48126-48137 (2017).

29. Wessely, F. \& Emes, R. D. Identification of DNA methylation biomarkers from Infinium arrays. Front. Genet. 3, 1-8 (2012).

30. Zheng, D. et al. Differentially methylated regions in patients with rheumatic heart disease and secondary pulmonary arterial hypertension. Exp. Ther. Med. 14, 1367-1372 (2017).

31. Peters, T. J. et al. De novo identification of differentially methylated regions in the human genome. Epigenetics Chromatin 8, 1-16 (2015).

32. Ramanan, V. K., Shen, L., Moore, J. H. \& Saykin, A. J. Pathway analysis of genomic data: concepts, methods, and prospects for future development. Trends Genet. 28, 323-332 (2012)

33. Olsson, M., Beck, S., Kogner, P., Martinsson, T. \& Carén, H. Genome-wide methylation profiling identifies novel methylated genes in neuroblastoma tumors. Epigenetics [Internet]. 11, 74-84 (2016).

34. Palmer, R. D. et al. Pediatric malignant germ cell tumors show characteristic transcriptome profiles. Cancer Res. 68, 4239-4247 (2008).

35. Brait, M. et al. DNA methylation profiles delineate epigenetic heterogeneity in seminoma and non-seminoma. Br. J. Cancer [Internet]. 106, 414-423 (2012).

36. Murray, M. J., Schönberger, S. in Pediatric Germ Cell Tumors: Biology of Germ Cell Tumors. First edn. (eds Frazier, A. L., Amatruda, J. F.) 1-15 (Springer-Verlag, Heidelberg New York Dordrecht London, 2014).

37. Luis, T. C. et al. Canonical wnt signaling regulates hematopoiesis in a dosagedependent fashion. Cell Stem Cell [Internet]. 9, 345-356 (2011).

38. Kato, N., Shibuya, H., Fukase, M., Tamura, G. \& Motoyama, T. Involvement of adenomatous polyposis coli (APC) gene in testicular yolk sac tumor of infants. Hum. Pathol. 37, 48-53 (2006).

39. Okpanyi, $V$ et al. Analysis of the adenomatous polyposis coli (APC) gene in childhood and adolescent germ cell tumors. Pediatr. Blood Cancer 56, 384-391 (2011).

40. Frazier, A. L., Amatruda, J. F. in Pediatric Germ Cell Tumors [Internet]. (eds Frazier, A. L., Amatruda, J. F.) 911-961 (Springer, Heidelberg New York Dordrecht London, 2009).

41. Schonberger, S. et al. Extracellular regulators of the Wnt signaling pathway in childhood germ cell tumors: Methylation of the SFRP2 promoter leads to Wnt activation and beta-catenin accumulation. Pediatr. Blood Cancer 55, 804-805 (2010).

42. Schuijers, J. et al. Ascl2 acts as an R-spondin/wnt-responsive switch to control stemness in intestinal crypts. Cell Stem Cell [Internet]. 16, 158-170 (2015).

43. $\mathrm{Wu}$, J. et al. Neuropeptide $\mathrm{Y}$ enhances proliferation and prevents apoptosis in rat bone marrow stromal cells in association with activation of the Wnt/ 3 -catenin pathway in vitro. Stem Cell Res. 21, 74-84 (2017).

44. Noor, D. A. M. et al. Genome-wide methylation analysis identifies genes silenced in non-seminoma cell lines. NPJ Genomic Med. 1, 15009 (2016).

45. Armstrong, C. L., Galisteo, R., Brown, S. A. N. \& Winkles, J. A. TWEAK activation of the non-canonical NF-kB signaling pathway differentially regulates melanoma and prostate cancer cell invasion. Oncotarget [Internet]. 7, 81474-81492 (2016).

46. Finkelstein, L. D., Shimizu, Y. \& Schwartzberg, P. L. Tec kinases regulate TCRmediated recruitment of signaling molecules and integrin-dependent cell adhesion. J. Immunol. [Internet]. 175, 5923-5930 (2005).

47. Middendorp, S., Dingjan, G. M., Maas, A., Dahlenborg, K. \& Hendriks, R. W. Function of Bruton's tyrosine kinase during B cell development is partially independent of its catalytic activity. J. Immunol. [Internet]. 171, 5988-5996 (2003).

48. Tang, X. -H. \& Gudas, L. J. Retinoids, retinoic acid receptors, and cancer. Annu Rev. Pathol. Mech. Dis. [Internet]. 6, 345-364 (2011).

49. Biankin, A. V. et al. Pancreatic cancer genomes reveal aberrations in axon guidance pathway genes. Nat. [Internet]. 491, 399-405 (2012).

50. Ju, J. A. \& Gilkes, D. M. RhoB: Team oncogene or team tumor suppressor?. Genes (Basel) [Internet]. 9, E67 (2018).

51. Curmi, P. A. et al. Overexpression of stathmin in breast carcinomas points out to highly proliferative tumours. Br. J. Cancer [Internet]. 82, 142-150 (2000). 
Differences in DNA methylation profiles by histologic subtype of...

LA Williams et al.

872

52. Draper, J. S. et al. Recurrent gain of chromosomes $17 q$ and 12 in cultured human embryonic stem cells. Nat. Biotechnol. 22, 53-54 (2004).

53. Kraggerud, S. M. et al. Genome profiles of familial/bilateral and sporadic testicular germ cell tumors. Genes Chromosome Cancer 34, 168-174 (2002).

54. Mostert, M. M. C. et al. Comparative genomic hybridization of germ cell tumors of the adult testis: Confirmation of karyotypic findings and identification of a $12 p$ amplicon. Cancer Genet. Cytogenet. 89, 146-152 (1996).

55. Von Eyben, F. E. Chromosomes, genes, and development of testicular germ cell tumors. Cancer Genet. Cytogenet. 151, 93-138 (2004).

56. Bussey, K. J. et al. Chromosomes 1 and 12 abnormalities in pediatric germ cell tumors by interphase fluorescence in situ hybridization. Cancer Genet. Cytogenet. 125, 112-118 (2001).

57. Pátja, K., Pukkala, E., Sund, R., livanainen, M. \& Kaski, M. Cancer incidence of persons with Down syndrome in Finland: A population-based study. Int. J. Cancer 118, 1769-1772 (2006).
58. Satgé, D. et al. An excess of testicular germ cell tumors in Down's syndrome: Three case reports and a review of the literature. Cancer [Internet]. 80, 929-935 (1997).

59. Aschim, E. L., Haugen, T. B., Tretli, S., Daltveit, A. K. \& Grotmol, T. Risk factors for testicular cancer - differences between pure non-seminoma and mixed seminoma/non-seminoma? Int. J. Androl. 29, 458-467 (2006).

60. Song, F. et al. Association of tissue-specific differentially methylated regions (TDMs) with differential gene expression. Proc. Natl Acad. Sci. [Internet]. 102, 3336-3341 (2005)

61. van Eijk, K. R. et al. Genetic analysis of DNA methylation and gene expression levels in whole blood of healthy human subjects. BMC Genomics 13, 636 (2012).

62. Michels, K. B. et al. Recommendations for the design and analysis of epigenomewide association studies. Nat. Methods 10, 949-955 (2013).

63. Oosterhuis, J. W. \& Looijenga, L. H. J. Testicular germ-cell tumors in a broader perspective. Nat. Rev. Cancer 5, 210-222 (2005). 